\title{
Solutions for modelling moving heat sources in a semi-infinite medium and applications to laser material processing
}

\author{
M. Van Elsen ${ }^{\mathrm{a}, *}$, M. Baelmans ${ }^{\mathrm{a}}$, P. Mercelis $^{\mathrm{a}}$ and J.-P. Kruth ${ }^{\mathrm{a}}$ \\ ${ }^{a}$ Departement of Mechanical Engineering, Catholic University of Leuven, \\ Celestijnenlaan 300b, B-3001 Heverlee, Belgium
}

\begin{abstract}
This study describes the analytical and numerical solution of the heat conduction equation for a localised moving heat source of any type for use in laser material processing, as welding, layered manufacturing and laser alloying. In this paper, the analytical solution for a uniform heat source is derived from the solution of an instantaneous point heat source. The result is evaluated numerically and is compared to existing solutions for the moving point source and a semi-ellipsoidal source. Next, the result is used to demonstrate how such model can be used to study the effect of the heat source geometry. Besides, this solution reveals that a melting efficiency higher than 0.37 (=1/e, a maximum value stated by Rykalin [1]) can be obtained. To investigate the effect of the temperature dependence of the material parameters, in particular the latent heat of fusion, a finite difference model is implemented. It is shown that the enthalpy method is most suited to implement the latent heat of fusion. A numerical evaluation for $\mathrm{TiAl}_{6} \mathrm{~V}_{4}$, reveals that the effect of the latent heat is rather small, except for the case when the conductivity is very low, e.g. when scanning in a loose powder bed. The results demonstrate that analytical and numerical solutions can be effectively used to calculate the temperature distribution in a semi-infinite medium for finite 3D heat sources. In this way, a tool to investigate the importance of different processing parameters in laser manufacturing is obtained.
\end{abstract}

Key words: Moving heat source, Semi-infinite body, Temperature distribution, Laser manufacturing

* Corresponding author. Tel.: +32-16-32-25-52; Fax: +32-16-32-29-87

Email address: Maarten.VanElsen@mech.kuleuven. be (M. Van Elsen). 


\begin{tabular}{|c|c|c|}
\hline Nomenclature & & \\
\hline$a_{h}, b_{h}, c_{h}, d_{h}$ & $m$ & Heat source parameters \\
\hline$A$ & - & Scaling factor \\
\hline$c_{a p p}$ & $J / k g K$ & Apparent heat capacity \\
\hline$c_{e f f}$ & $J / k g K$ & Effective heat capacity \\
\hline$c_{L_{f}}$ & $J / k g K$ & Heat capacity due to latent heat of fusion \\
\hline$c$ & $J / k g K$ & Heat capacity \\
\hline$d_{x}$ & $m$ & Spot size along $\mathrm{x}$ \\
\hline$d_{y}$ & $m$ & Spot size along y \\
\hline Fo & - & Fourier number \\
\hline$h$ & $J / k g$ & Enthalpy \\
\hline$k$ & $W / m K$ & Conductivity \\
\hline$L_{f}$ & $J / k g$ & Latent heat of fusion \\
\hline$n$ & - & Operating parameter \\
\hline$P_{L}$ & $W$ & Laser power \\
\hline$P e$ & - & Peclet number \\
\hline$\dot{q}$ & $W / m^{3}$ & Volumetric heat source \\
\hline$\dot{q}_{L_{f}}$ & $W / m^{3}$ & Volumetric heat source due to $L_{f}$ \\
\hline$R$ & $m$ & Radial distance \\
\hline$r$ & $m$ & Radius \\
\hline$S$ & $m^{2}$ & Cross section area \\
\hline$t$ & $s$ & Time \\
\hline$T$ & $K$ & Temperature \\
\hline$T_{0}$ & $K$ & Room temperature \\
\hline$T_{l}$ & $K$ & Liquidus temperature \\
\hline$T_{m}$ & $K$ & Melting temperature \\
\hline$T_{s}$ & $K$ & Solidus temperature \\
\hline$u$ & $J / k g$ & Internal energy \\
\hline$V$ & $m / s$ & Scan speed \\
\hline$x$ & $m$ & Coordinate along scanning direction \\
\hline$y$ & $m$ & Coordinate perpendicular to scanning direction \\
\hline$z$ & $m$ & Coordinate in depth \\
\hline
\end{tabular}




\begin{tabular}{|lll|}
\hline Nomenclature & & \\
$\delta h$ & $\mathrm{~J} / \mathrm{kg}$ & Melt enthalpy \\
$\kappa$ & $\mathrm{m}^{2} / \mathrm{s}$ & Thermal diffusivity \\
$\lambda$ & $m$ & Wavelength of error term \\
$\rho$ & $\mathrm{kg} / \mathrm{m}^{3}$ & Density \\
$\sigma$ & $(v a r)$ & Standard deviation \\
$\theta$ & - & Dimensionless temperature \\
\hline
\end{tabular}

\section{Introduction}

All laser material processing techniques require the correct amount of energy at the right place and at the right time to ensure successful processing. The thermal history of the process influences the melt pool behaviour, thermal stresses, microstructure, etc. As such, it determines the final properties of the processed part as strength, elongation, fatigue behaviour, hardness and accuracy.

Therefore, it is of crucial importance to acquire reliable and accurate predictions of the physical effects that occur during processing. Indeed, mathematical models are used to gain insight in the process, as experiments are rather expensive. It is for example difficult to test different kinds of laser beams, simply because for every test, new equipment (laser and/or optics) is needed. Indeed, the laser beam energy profile cannot be modified freely. As a typical example, before installing a dual beam scanning system, it can be interesting to investigate the effect of such configuration on the temperature distribution.

Throughout this paper, the effect of different localised 3D moving heat sources on a semi-infinite medium with variable material properties is investigated, assuming there is only conductive heat transport. The major drawback lies in the fact that the behaviour of molten material cannot be taken into account.

Different authors, as Rosenthal [2] and Rykalin [3], have studied classical solutions of the heat conduction equations. Carslaw and Jaeger [4] have brought this together in a complete reference book with analytical solutions of the heat conduction equation. The heat sources used are point sources, line sources and plane sources, since these are the only types of geometry were analytical solutions are straightforward to obtain. This type of sources is suited to predict the thermal history at a large distance from the source. However, they are useless near the heat source, since temperature in the origin would become infinite. To overcome this problem, 2D heat sources were introduced by Eager 
and Tsai [5]. The first to introduce a 3D heat source, was Goldak et al. [6]. He used a double ellipsoidal moving heat source to calculate the temperature field with finite element modelling. Especially for the prediction of deeper welds, this was an improvement compared to 2D heat sources. More recently Nguyen et. al. $[7,8]$ developed a closed form analytical solution for this kind of 3D heat sources in a semi-infinite body or in a thick plate. This enabled them to predict the melt pool geometry. Further, many more authors have used numerical techniques to evaluate particular problems in heat transfer. A review is given by $\mathrm{Hu}$ and Argyropoulos [9].

In the field of Rapid Manufacturing, many authors describe the modelling of a specific process, with specific boundary conditions, by use of FEM software ( $[10,11]$, a.o.). Amongst them, Chen and Zhang [12] have written an interesting contribution on the modelling of the shrinkage during processing of powder mixtures. Besides, some authors have described models for very specific subprocesses that occur during SLM. Gusarov and Kruth [13] have described the absorptivity of lasers used on powder beds. Determination of the contact angle of a fluid on a solid is described by Gould [14]. Roy and Schwartz have described the stability of liquid ridges [15]. Modelling of marangoni convection is less straightforward.

The aim of the present study is to investigate the relative importance of different parameters for laser manufacturing. If the effect of the heat source is to be studied, an analytical solution can be effective. If the effect of material properties is to be studied, the use of a finite difference model (FDM) is obvious. This paper contains the mathematical exposition, needed for these investigations. The paper is organised as follows: section 2 contains the mathematical relations that describe the general process of heat transfer. In section 3 , these equations, are solved analytically for different heat source geometries and constant material parameters. In section 4, a FDM for a 3D semi-infinite medium is derived. Different techniques to implement the latent heat of fusion are discussed.

\section{Heat conduction equation for moving heat sources}

The general convection-diffusion equation can be written as

$$
\frac{\partial \rho u}{\partial t}+\frac{\partial \rho h V}{\partial x}=\nabla \cdot(k \nabla T)+\dot{q}
$$

where $u$ is the internal energy, $h$ the enthalpy, $\rho$ the density, $k$ the conductivity, $\dot{q}$ a volumetric heat source, $T$ the temperature and $V$ the speed of either the heat source or the medium. In most applications, the origin of the coordinate system is fixed to the centre of the heat source on top of the processed surface. 
The $x$ direction corresponds to the constant speed of a moving heat source. $y$ is the direction perpendicular to $x$, in the plane of the processed material surface, and $z$ is directed inside the processed material. The first term in eq. (1) on the left hand side represents the change of internal energy and the second is a convective term. On the right hand side, there is the conductive term and a heat source or sink. For $V=0$, this equation becomes the heat conduction equation, given that $d u=c d T$, with $c$ the heat capacity:

$$
c \frac{\partial \rho T}{\partial t}=\nabla \cdot(k \nabla T)+\dot{q}
$$

The steady state equation with constant velocity $V$, can be simplified using the continuity equation

$$
\frac{\partial \rho}{\partial t}+\frac{\partial \rho V}{\partial x}=0
$$

resulting in

$$
\rho c(T) V \frac{\partial T}{\partial x}=\nabla \cdot(k(T) \nabla T)+\dot{q}
$$

given that $d u=d h=c d T$.

\section{Analytical solution of the heat conduction equation}

In this section, the analytical solution of a uniform heat source, irradiating a semi-infinite 3D medium is derived. The temperature field will be compared to existing analytical solutions.

Firstly, the well known solution of a point heat source is given as a reference. Secondly, the solution of a semi-ellipsoidal moving heat source is given (Goldak et al. [6]). And thirdly, the solution for a uniform finite heat source is deduced. Subsequently, these results are used to study the effect of the heat source geometry on the temperature field. First, it is shown for a numerical example that near the heat source, the temperature field is different for different heat sources. Next, the consequences of this for the melting efficiency of a process are discussed. Further, it is shown that one can predict the temperature field of very complex heat sources. Finally, the results are used to get some insight in what happens if the Peclet number is changed. This is important when studying the effect of the latent heat, a topic that will be discussed in more details in section 4 . 


\subsection{Moving point heat source}

The derivation and the analytical solution of the temperature field induced by a moving point heat source in a 3D semi-infinite body is a.o. described by Carslaw and Jaeger [4]:

$$
\theta=\frac{P_{L}}{4 \pi k R\left(T_{m}-T_{0}\right)} \exp (-V(R+x) / 2 \kappa)
$$

with the dimensionless temperature $\theta=\left(T-T_{0}\right) /\left(T_{m}-T_{0}\right), \kappa$ the thermal diffusivity, defined as $\kappa=\frac{k}{\rho c}, P_{L}$ the applied laser power and $R$ the distance from the heat source location $\left(R^{2}=x^{2}+y^{2}+z^{2}\right)$.

From this equation, it is clear that the temperature at the heat source location is infinite. Although this equation cannot accurately predict the temperature field in the neighbourhood of a finite heat source, it can serve well for validation purposes. Indeed, far from the source, the isotherms should tend to coincide. It should be noted here that calculating the temperature field of a heat source scanning a line in a loose powder bed, as is done in Selective Laser Sintering and Selective Laser Melting [16,17], is not possible analytically. The reason is that part of the material will remain powder, and part of the material will be converted to liquid material and, after solidification, to solid material. This gives rise to huge differences in the temperature field, due to large differences in thermal conductivity.

\subsection{Semi-ellipsoidal moving heat source}

Goldak et al. [6] were the first to introduce a 3D heat source, more in particular a semi-ellipsoidal heat source, with heat flux $\dot{q}$ :

$$
\dot{q}(x, y, z)=\frac{6 \sqrt{3} P_{L}}{a_{h} b_{h} c_{h} \pi \sqrt{\pi}} \exp \left(-\frac{3 x^{2}}{c_{h}^{2}}-\frac{3 y^{2}}{a_{h}^{2}}-\frac{3 z^{2}}{b_{h}^{2}}\right)
$$

with $a_{h}, b_{h}$ and $c_{h}$ heat source geometry parameters.

The solution for the temperature field in a semi-infinite body was derived by NGuyen et al. [7]. The result in dimensionless form is given as

$$
\frac{\theta}{n}=\frac{1}{\sqrt{2 \pi}} \int_{0}^{\frac{V^{2} t}{2 \kappa}} \frac{d \tau}{\sqrt{\tau+u_{a}^{2}} \sqrt{\tau+u_{b}^{2}}}\left(\frac{A_{1}}{\sqrt{\tau+u_{c}^{2}}}\right)
$$


where

$$
A_{1}=\exp \left(-\frac{(\xi+\tau)^{2}}{2\left(\tau+u_{c}^{2}\right)}-\frac{\psi^{2}}{2\left(\tau+u_{a}^{2}\right)}-\frac{\zeta^{2}}{2\left(\tau+u_{b}^{2}\right)}\right)
$$

The dimensionless parameters are defined as recommended by Christensen's method [18]: $\xi=V x / 2 \kappa, \psi=V y / 2 \kappa, \zeta=V z / 2 \kappa, \tau=V^{2}\left(t-t^{\prime}\right) / 2 \kappa, u_{a}=$ $V a_{h} 2 \sqrt{6} \kappa, u_{b}=V b_{h} 2 \sqrt{6} \kappa, u_{c}=V c_{h} 2 \sqrt{6} \kappa$ and $n=P_{L} V /\left(4 \pi \kappa^{2} \rho c\left(T_{m}-T_{0}\right)\right)$.

This solution approximates the temperature field near the laser spot fairly well in most cases, since normally, the beam profile resembles quite good this type of source, except that a real heat source does not spread to infinity, as is the case for a Gaussian profile.

Goldak et al. experienced that this type of heat source predicted the temperature gradients in front of the source to be less steep than experimentally observed. Therefore, they created the double ellipsoidal heat source, which is a mathematical deformation of the semi-ellipsoidal or ellipsoidal heat source. However, in practice, most heat sources are symmetrical. In this paper, artificial heat sources will not be used, because for the purpose of this research, this seems of little value.

On the other hand, the fact that a small change of the source intensity profile results in a noticeable change in temperature field, is an indication that the heat source geometry is significant for the temperature field.

In order to study the effect of source shapes, the solution of the ellipsoidal heat source is not very useful, because it is not straightforward to create other geometries by adding multiple ellipsoidal heat sources. As a basis for complex heat source geometries, a heat source that is completely uniform is optimal. Therefore, the solution for a uniform moving heat source will be deduced in the next section.

\subsection{Uniform moving heat source}

The heat flux $\dot{q}(x, y, z)$ at a point $(x, y, z)$ within a uniform source is given by:

$$
\dot{q}(x, y, z)=\frac{P_{L}}{4 a_{h} b_{h} c_{h}} \quad\left\{\begin{array}{l}
-c_{h}<x<c_{h} \\
-a_{h}<y<a_{h} \\
0<z<b_{h}
\end{array}\right.
$$

As for the other types of heat sources, the solution is based on the solution for an instantaneous point source in fixed coordinates [4]: 


$$
\begin{aligned}
d T_{t^{\prime}}= & \frac{\dot{q} d t^{\prime}}{\rho c\left[4 \pi \kappa\left(t-t^{\prime}\right)\right]^{3 / 2}} \\
& \exp \left(-\frac{\left(x-x^{\prime}\right)^{2}+\left(y-y^{\prime}\right)^{2}+\left(z-z^{\prime}\right)^{2}}{4 \kappa\left(t-t^{\prime}\right)}\right)
\end{aligned}
$$

where $\left(x^{\prime}, y^{\prime}, z^{\prime}\right)$ is the location of the instant point source and $d T_{t^{\prime}}$ is the transient temperature increase at the observation time $t$ due to the point heat source $\dot{q}$ at time $t^{\prime}$.

Integration over the volume of the heat source and substitution of eq. (8) gives:

$$
\begin{aligned}
d T_{t^{\prime}}= & \int_{-c_{h}}^{c_{h}} d x^{\prime} \int_{-a_{h}}^{a_{h}} d y^{\prime} \int_{-b_{h}}^{b_{h}} d z^{\prime} \frac{d t^{\prime}}{\rho c\left[4 \pi \kappa\left(t-t^{\prime}\right)\right]^{3 / 2}} . \\
& \frac{P_{L}}{4 a_{h} b_{h} c_{h}} \exp \left(-\frac{\left(x-x^{\prime}\right)^{2}+\left(y-y^{\prime}\right)^{2}+\left(z-z^{\prime}\right)^{2}}{4 \kappa\left(t-t^{\prime}\right)}\right)
\end{aligned}
$$

The integrals can be calculated as follows:

$$
\begin{aligned}
I & =\int_{-c_{h}}^{c_{h}} \exp \left(-\frac{\left(x-x^{\prime}\right)^{2}}{4 \kappa\left(t-t^{\prime}\right)}\right) d x^{\prime} \\
& =-\sqrt{4 \kappa\left(t-t^{\prime}\right)} \int_{-c_{h}}^{c_{h}} \exp \left(-\frac{\left(x-x^{\prime}\right)^{2}}{4 \kappa\left(t-t^{\prime}\right)}\right) d\left(\frac{x-x^{\prime}}{\sqrt{4 \kappa\left(t-t^{\prime}\right)}}\right) \\
& =-\frac{\sqrt{4 \kappa \pi\left(t-t^{\prime}\right)}}{2}\left(\operatorname{Erf}\left(\frac{x-c_{h}}{\sqrt{4 \kappa\left(t-t^{\prime}\right)}}\right)-\operatorname{Erf}\left(\frac{x+c_{h}}{\sqrt{4 \kappa\left(t-t^{\prime}\right)}}\right)\right)
\end{aligned}
$$

With $F_{o_{s}}$ the Fourier number based on $s$ and $t-t^{\prime}$ as length and time respectively, $\operatorname{Erfh}\left(x, s, t^{\prime}\right)$ is defined as

$$
\begin{aligned}
\operatorname{Erfh}\left(x, s, t^{\prime}\right) & \triangleq \operatorname{Erf}\left(\frac{x-s}{\sqrt{4 \kappa\left(t-t^{\prime}\right)}}\right)-\operatorname{Erf}\left(\frac{x+s}{\sqrt{4 \kappa\left(t-t^{\prime}\right)}}\right) \\
& =\operatorname{Erf}\left(\frac{\sqrt{F o_{s}}}{2}\left(\frac{x}{s}-1\right)\right)-\operatorname{Erf}\left(\frac{\sqrt{F o_{s}}}{2}\left(\frac{x}{s}+1\right)\right) \\
& \triangleq \operatorname{Erfh}\left(\frac{x}{s}, F o_{s}\right)
\end{aligned}
$$

and substituting eqs. (11) and (12) in eq. (10) results in: 


$$
d T_{t^{\prime}}=-\frac{P_{L} d t^{\prime}}{2^{5} \rho c a_{h} b_{h} c_{h}} \operatorname{Erfh}\left(x, c_{h}, t^{\prime}\right) \operatorname{Erfh}\left(y, a_{h}, t^{\prime}\right) \operatorname{Erfh}\left(z, b_{h}, t^{\prime}\right)
$$

Integration over time gives

$$
\begin{aligned}
T-T_{0}= & -\frac{P_{L}}{2^{5} \rho c a_{h} b_{h} c_{h}} \int_{0}^{t} \operatorname{Erfh}\left(x+V\left(t-t^{\prime}\right), c_{h}, t^{\prime}\right) \\
& \operatorname{Erfh}\left(y, a_{h}, t^{\prime}\right) \operatorname{Erfh}\left(z, b_{h}, t^{\prime}\right) d t^{\prime}
\end{aligned}
$$

for a uniform heat source moving with velocity $V$ along the $x$ direction.

The heating efficiency is defined as $\eta=\frac{\rho c a_{h} b_{h} c_{h}\left(T_{m}-T_{0}\right)}{P_{L} t}$. The latter is closely related to the melting efficiency which will be used later. The equation can now be formulated dimensionless:

$$
\begin{gathered}
\theta=-\frac{1}{2^{5} \eta} \int_{0}^{t} \operatorname{Erfh}\left(\frac{x+V\left(t-t^{\prime}\right)}{c_{h}}, F o_{c_{h}}\right) \\
\operatorname{Erfh}\left(\frac{y}{a_{h}}, F o_{a_{h}}\right)\left(\frac{z}{b_{h}}, F o_{b_{h}}\right) \frac{d t^{\prime}}{t}
\end{gathered}
$$

$\theta(x, y, z, t)$ can be evaluated by numerical integration. For $t \rightarrow \infty$ or $F_{O} \rightarrow$ $\infty$, we obtain a steady state situation. Practically, for most cases in laser processing, already after $1 \mathrm{~s}$, the steady state situation has settled.

Another important dimensionless parameter in heat transfer is the Peclet number $P e$, which measures the proportion of convection to conduction. $P e$ is defined in this paper as:

$$
P e=\frac{V c_{h}}{\kappa}
$$

For a given heat source, the steady state temperature field can now be written in function of $P e$ and $\eta$, where the latter one only results in a scaling of the temperature field, as can be seen from eq. (15). Because $P e$ is often used as a reference, it will be mentioned for each example. The shape of the temperature field will also depend on the type of heat source, as will be shown in the next section.

\subsection{Numerical evaluation}

All equations are implemented in Matlabß, using a trapezium rule for the numerical integration over time. The material parameters used in this study 
correspond to that of $\mathrm{TiAl}_{6} \mathrm{~V}_{4}: T_{m}=1933 \mathrm{~K}, T_{0}=293 \mathrm{~K}, \rho=4450 \mathrm{~kg}$, $c=564 \mathrm{~J} / \mathrm{kgK}, k=6 \mathrm{~W} / \mathrm{mK}$ (resulting in a diffusivity $\kappa=2.4 \cdot 10^{-6} \mathrm{~m}^{2} / \mathrm{s}$ ). The time to obtain steady state was chosen to be $t=1.5 \mathrm{~s}$. A conductivity of $6 \mathrm{~W} / \mathrm{mK}$, slightly lower than normal bulk $\mathrm{TiAl}_{6} \mathrm{~V}_{4}$, was used because parts produced by selective laser melting often have still some amount of porosity, which lowers the conductivity [19-22].

Figure 1a-c gives the result for a scanning speed $V=0.05 \mathrm{~m} / \mathrm{s}$, laser power $P_{L}=25 \mathrm{~W}, c_{h}=a_{h}=0.15 \mathrm{~mm}, b_{h}=0.05 \mathrm{~mm}$ (thus $P e=3.14$ ) and this for $z=0$, which is on top of the solid. For the point heat source, the isothermal lines are omitted for values higher than 1.6. At $(0,0,0)$ the temperature in this case is infinity.

The figures confirm intuitive trends: the more the energy is concentrated, the higher the peak temperature. Higher temperatures, for a constant energy density $P_{L} / V$, can be obtained by choosing a source geometry with a higher energy flux near the centre or by decreasing the spot size. The location of the peak temperature is situated near the rear end of the spot. The higher the speed of the heat source, the more the peak temperature is situated at the rear end. However, it lies always inside the spot. The ellipsoidal heat source does not has an edge, but most of the energy is delivered inside the so-called spot. Usually, the spot is defined as the region where the intensity amounts to $1 / e^{2}$ or higher (see [23] a.o.).

A comparison between the different plots reveals that far from the origin, the isothermal lines almost match, which is in agreement with the expectations. However, in order to achieve a reliable estimate of the peak temperature or an estimate of the thermal gradients near the spot, a good choice of the heat source is necessary.

\subsection{Melting efficiency}

The resulting temperature field of a uniform moving heat source, can be used to comment on the idea of the melting efficiency.

For laser processes where melting occurs, the melting efficiency is defined as the ratio of the minimal amount of energy needed to warm up and melt a given amount of material, compared to the added energy.

$$
\eta_{m}=\frac{V S \delta h}{P_{L}}
$$

where $S$ is the cross section area of the molten track. Often $S$ is approximated as the width of the melt pool multiplied by the depth of the melt pool. Heat 


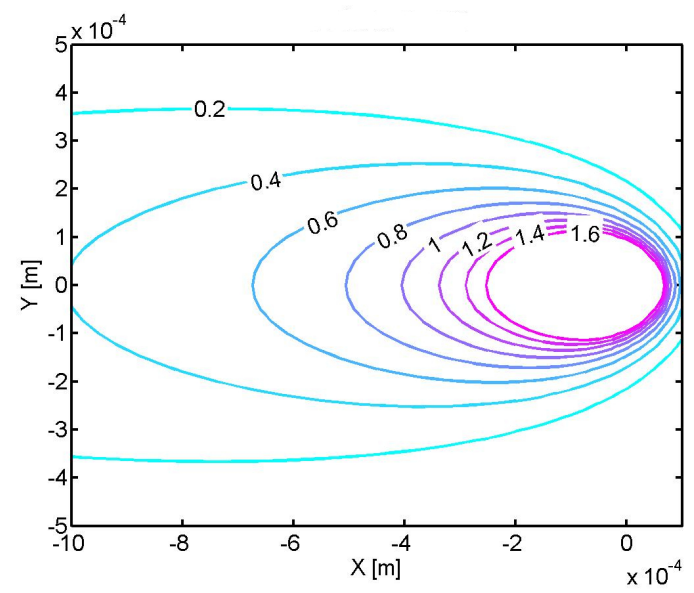

a) Point heat source

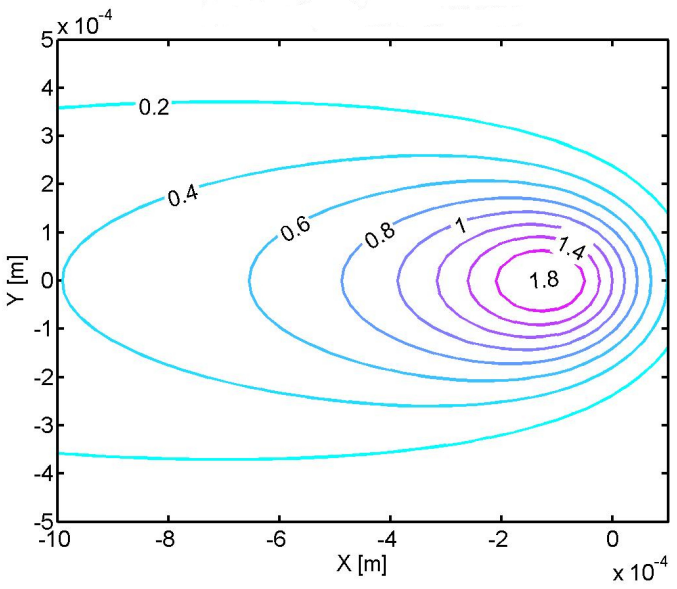

b) Ellipsoidal heat source

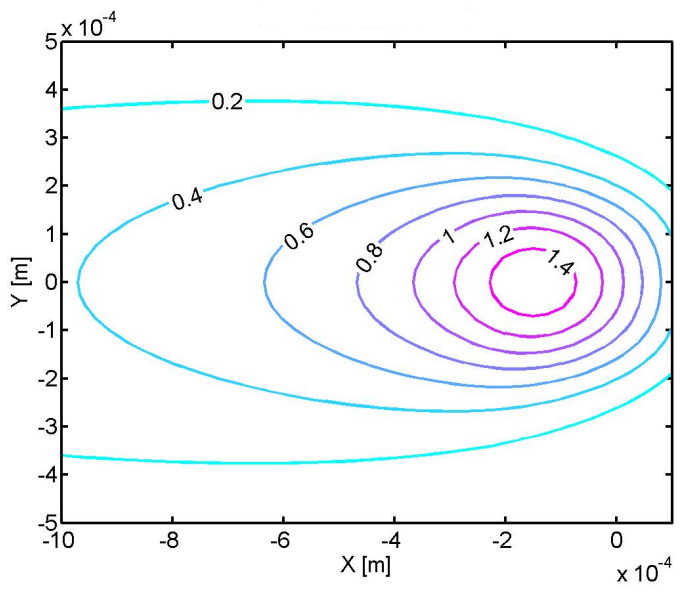

c) Uniform heat source

Fig. 1. Temperature field for various heat sources 
that is not used for melting, is used for overheating of the melt pool or is lost by conduction. The latter results in the heating of the material next to the molten track ${ }^{1}$. It should be noted that different heat source geometries will result in different values of $\eta_{m}$.

Rykalin [3] derived that the maximum melting efficiency for a point heat source is about 0.37 for the $3 \mathrm{D}$ case. However, does this mean that in practice no higher efficiencies can be achieved? There are at least two ways to get a higher efficiency, in theory.

In general, high efficiencies can be obtained for high scanning speeds, since then, the conduction can be neglected. In the limit, no energy is lost by conduction, since conduction needs time. A simulation of a stationary point heat source, reveals that in the very beginning, all the excess of energy is found in the overheating of the melt. Indeed, for a point heat source, there will always be overheating of the melt since the peak temperature is infinite. The simulation suggests that overheating is the reason for the limited melting energy.

On the other hand, suppose now that a uniform source is used, that has just enough energy to melt part of the material, with hardly any overheating. Suppose, the conductivity is low and the scanning speed is very high. Intuitively, it is expected that the melting efficiency is 1 . From a mathematical point of view, however, it is not easy to prove this. Therefore, the integration is performed numerically. Parameters are $V=2 \mathrm{~m} / \mathrm{s}, P_{L}=125 \mathrm{~W}, k=0.1 \mathrm{~W} / \mathrm{mK}$, $t=0.1 \mathrm{~s}, a_{h}=c_{h}=0.15 \mathrm{~mm}, b_{h}=0.05 \mathrm{~mm}$, resulting in $P e=7529$. The resulting temperature field is given in figure 2. The depth of the melt pool is about $0.042 \mathrm{~mm}$ and the efficiency is about 0.67. Going faster or decreasing the conductivity, will increase the efficiency. In fact, the higher $P e$, the higher the efficiency.

Another possibility to increase the efficiency is the fact that part of the energy can be recycled. This idea is only significant for rapid prototyping techniques, where large surfaces are scanned. Suppose one wants to scan a circular surface and starts from the outer edge. The first scan is a fast circle with high efficiency. The second scan, a smaller circle is made some seconds later, and so on. Some heat will be conducted to the inner part of the circle. For the last scan, the part will have been preheated significantly and therefore, the laser power can be decreased, increasing the efficiency. The same is seen when scanning geometries with sharp edges in a loose power bed. The scanning parameters should be adapted to compensate for the heat accumulation, which is in fact a recuperation of already used heat.

1 In addition, energy is lost by radiation, by convection with the surrounding at-
mosphere and by expulsion of material. These effects are not taken into account in
this simple model. However, in a lot of relevant cases, these losses are negligible [24]. 


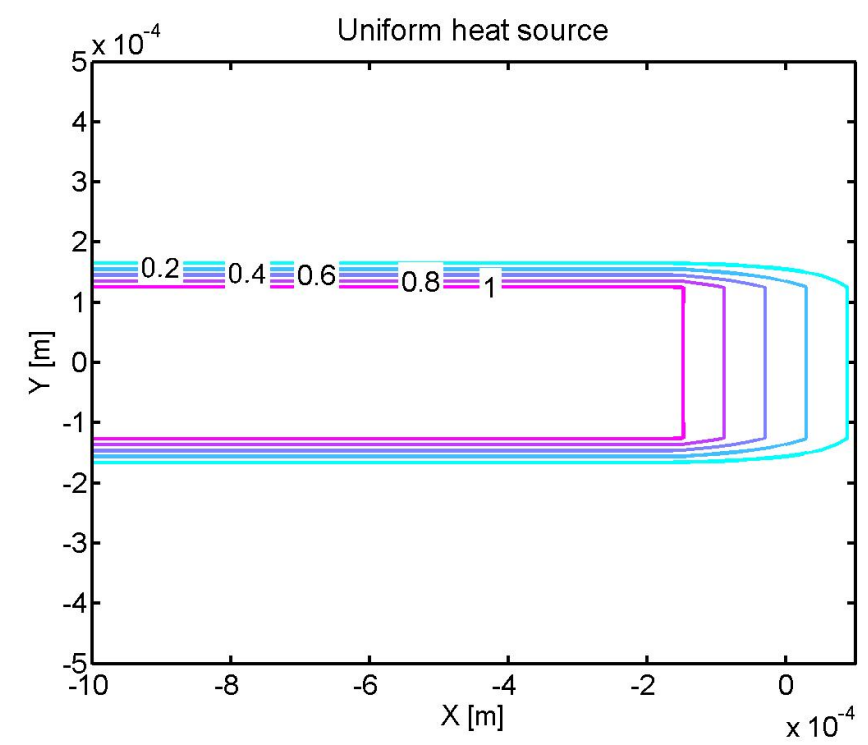

Fig. 2. Temperature field for high speed scanning with a uniform heat source $(P e=7529)$

A problem with the uniform heat source is that it is not axi-symmetric. Thus, only scanning in $x$ and $y$ direction will give the maximum efficiency. An axial symmetrical source should be circular with a lower heat flux in the centre, to avoid overheating.

A simulation reveals that, in the $2 \mathrm{D}$ case (in practice, we do not have any control on the third dimension), a parabolic heat source will have very good characteristics to obtain a high efficiency. Consider a heat source with intensity $I$ and radial distance $r$ :

$$
I(r)=A\left(r^{2}+d_{h}\right)
$$

with $A$ a scaling factor that scales with the total power. For a beam with diameter $d_{x}$, an offset $d_{h} \approx d_{x} / 2$ gives the wanted effect. This is shown in figure 3 for a beam diameter $d_{x}=2$ and $A=1$. The decrease at the edges in total added energy is very steep, and in the middle, there is somewhat less energy than further to the sides. This is wanted, because there will be time for conduction and convection, and the middle of the melt pool will actually heat up. For an offset of $0.75 d_{x}$, the delivered energy is quasi uniform till a radius of $0.7 d_{x}$.

We conclude that in theory the melting efficiency can be higher than the value stated by Rykalin. In general, a donut-shaped heat source will be best to get a higher efficiency. 


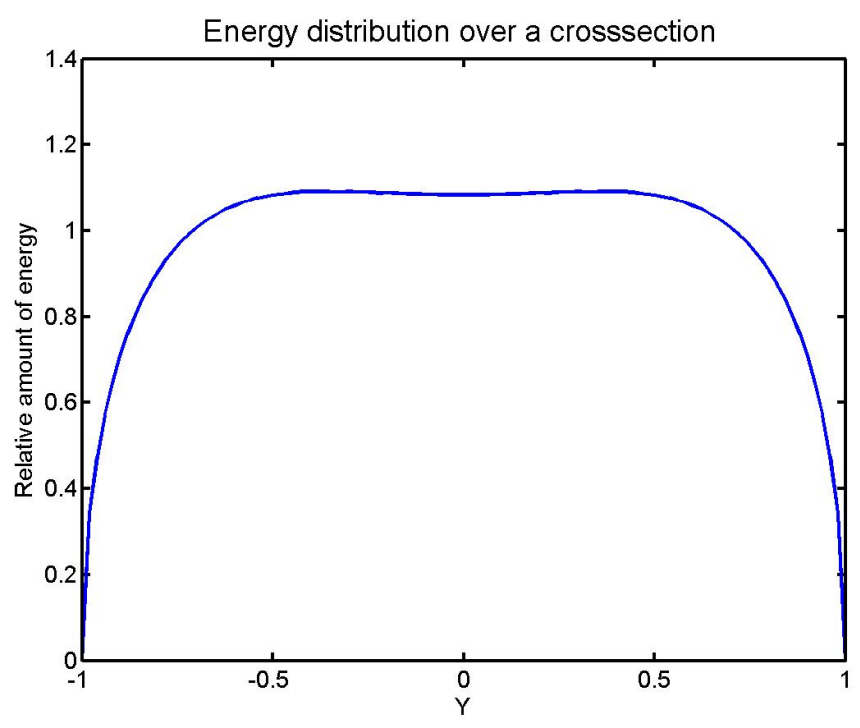

Fig. 3. Energy distribution through the cross section of a parabolic heat source

\subsection{Other source geometries}

The heat conduction equation is linear. Therefore, the solution of this equation for two heat sources is the same as the sum of the solutions of the separate heat sources. Thus, whatever geometry that is wanted, can be obtained by a linear combination of elementary heat sources. As an example, this was implemented for a 'smiley' (figure 4, left), consisting of 2 semi-ellipsoidal heat sources with $a_{h}=c_{h}=0.15 \mathrm{~mm}$, and $P_{L}=20 \mathrm{~W}$ (the eyes) and 6 uniform heat sources with parameters $a_{h}=c_{h}=0.075 \mathrm{~mm}$ and $P_{L}=10 \mathrm{~W}$ (the mouth). $b_{h}=0.05$ for all sources. The other parameters are $k=6 \mathrm{~W} / \mathrm{mK}$ and $V=0.05 \mathrm{~m} / \mathrm{s}$. The result is given in figure 4 on the right ${ }^{2}$.

\subsection{Latent heat of fusion}

Analytically, it is impossible to take into account the effect of the latent heat. Nevertheless, the latent heat has a significant effect on the melt pool size and the melt pool shape. As explained by De Lange [25], in the zone were the solidification occurs, the Peclet number becomes very high because of $L_{f}$, reducing the importance of the conduction, thus making the rear end of the melt isotherm much sharper than the other isothermal lines. This effect can be seen by simulating the temperature field for different samples of homogenous

$\overline{2}$ The reason why this irrelevant source geometry is chosen, lies in the fact that the visual effect for such a source, shows the difference more clearly. The isotherms for a round source, always have the same shape. 

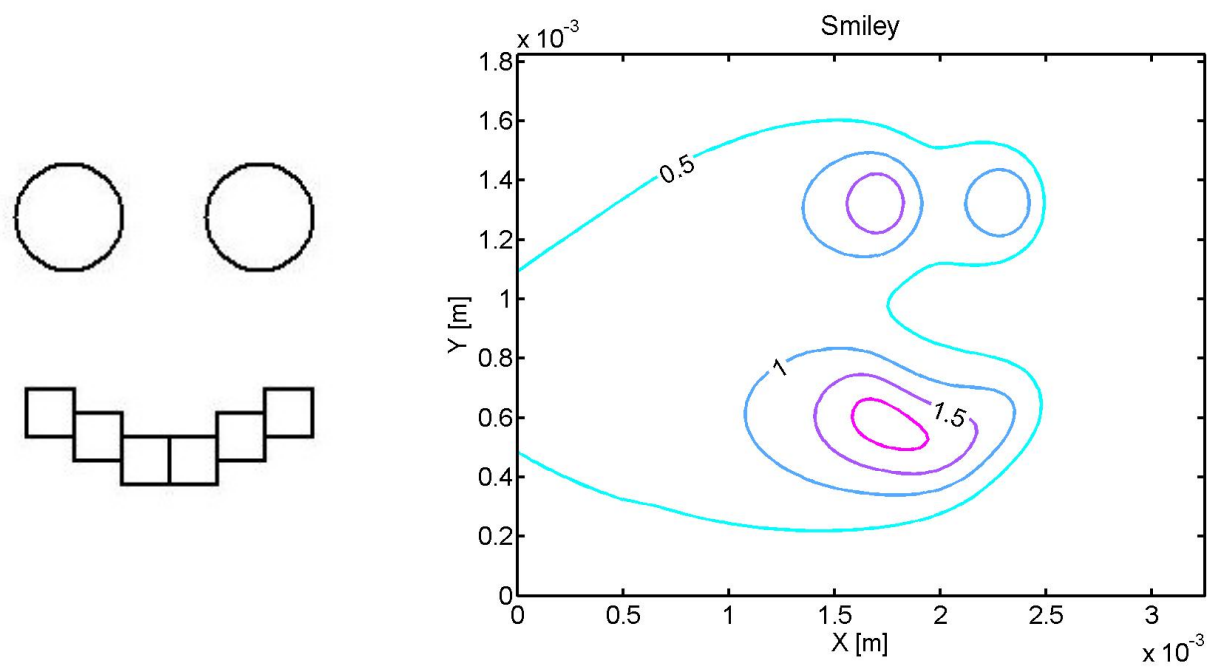

Fig. 4. Temperature field of a 'Smiley'

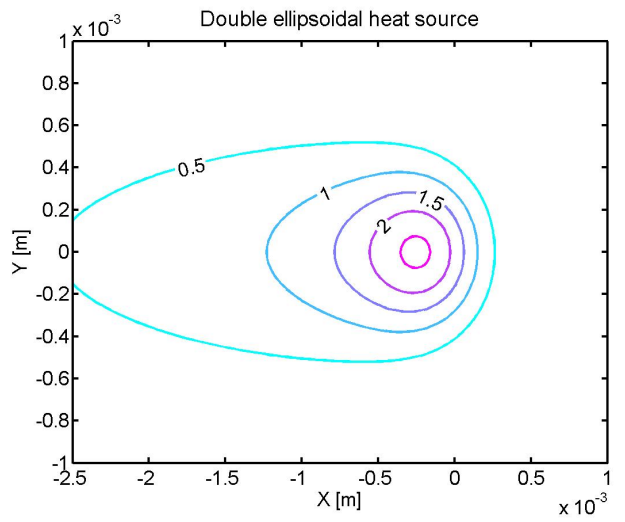

a) $k=7 \mathrm{~W} / \mathrm{mK}$ and $P_{L}=100 \mathrm{~W}$

$P e \approx 9$

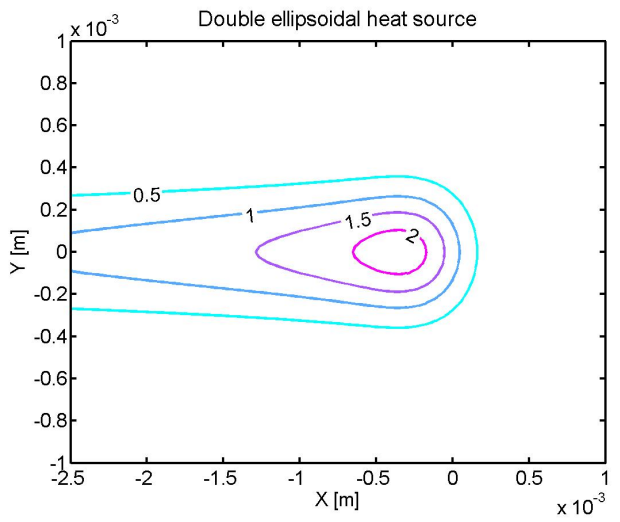

b) $k=0.1 \mathrm{~W} / \mathrm{mK}$ and $P_{L}=10 \mathrm{~W}$

$P e \approx 625$

Fig. 5. Effect of Pe on the shape of the isotherms

materials with different, but constant Peclet numbers. The result is given in figure 5 , with parameters $a_{h}=c_{h}=0.5 \mathrm{~mm}, b_{h}=0.05 \mathrm{~mm}$ and $V=0.05 \mathrm{~m} / \mathrm{s}$.

The result can be understood easily: if the temperature gradient after the laser becomes smaller, as certainly is the case for the region around melting temperature, the importance of the heat conduction perpendicular to the moving direction gains in importance, thus changing the isothermal lines. 


\section{Finite difference model}

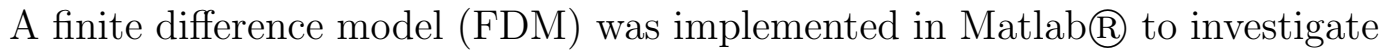
the effect of the material parameters temperature dependence and in particular the effect of the latent heat, since simulations have shown that the effect is significant.

\subsection{Derivation of the model}

Time-marching or false time stepping is used to make the convection-diffusion equation easier to solve. We are only interested in the steady state solution. The equation now becomes

$$
\rho c\left(T^{\star}\right) \frac{\partial T}{\partial t}+\rho c(T) V \frac{\partial T}{\partial x}=\nabla \cdot(k(T) \nabla T)+\dot{q}
$$

for a heat source moving to the left. $T^{\star}$ is a fixed temperature, that can be chosen freely at the beginning. The exact value will not influence the steady state solution. It should be noted here, that for a transient temperature field, modelled with a fixed grid, $\rho$ should be constant in order to conserve mass. However, for a steady state solution in a semi-infinite medium, this requirement should not be fulfilled.

Implicit Euler is used for the time integration:

$$
\Phi^{n+1}-\Phi^{n}=f\left(t_{n+1}, \Phi^{n+1}\right) \Delta t
$$

where $\Phi=T$ in our case.

For the convective term, a first order upwind difference scheme is used. For $V>0$, this becomes:

$$
\rho c(T) V \frac{\partial T}{\partial x} \rightarrow V \rho_{i} \frac{c\left(T_{i}\right)+c\left(T_{i-1}\right)}{2}\left(\frac{T_{i}-T_{i-1}}{x_{i}-x_{i-1}}\right)
$$

For the diffusive term, a central difference scheme is used

$$
\begin{aligned}
\nabla \cdot(k(T) \nabla T) \rightarrow & \left(k_{i j h}+k_{i+1}\right)\left(\frac{T_{i+1}-T_{i j h}}{\left(x_{i+1}-x_{i j h}\right)\left(x_{i+1}-x_{i-1}\right)}\right)- \\
& \left(k_{i j h}+k_{i-1}\right)\left(\frac{T_{i j h}-T_{i-1}}{\left(x_{i j h}-x_{i-1}\right)\left(x_{i+1}-x_{i-1}\right)}\right)+\ldots
\end{aligned}
$$


The material parameters are evaluated at moment $t$. For each time step, the equation can be written as

$$
A T^{t+1}=B T^{t}+\dot{q}
$$

\subsection{Boundary conditions}

There are 2 different kinds of boundary conditions that can be used. The first one is a known temperature on the boundary. The second one is a known heat flux. The latter is used to incorporate the symmetry boundary conditions, where the heat flux is zero perpendicular on that face.

The advantage of the analytical solution is that one can calculate the temperature at a particular point, without calculating the complete temperature field. Therefore, using the analytical solution as boundary condition, is fast and easy to implement. In this study, the Goldak heat source is used. This way, the size of the grid can be limited, because room temperature is only found far from the origin.

\subsection{Stability and convergence}

Define $d$ as the inverse of a Peclet number and $\nu$ as a dimensionless speed so that

$$
d=\frac{k \Delta t}{\rho c\left(T^{\star}\right) \Delta x^{2}}
$$

and

$$
\nu=\frac{V \Delta t}{\Delta x} \frac{\rho c}{\rho c\left(T^{\star}\right)}
$$

Then the footprint becomes, as described by Hirsch [26],

$$
\begin{aligned}
f(\lambda) \Delta t & =(-2 d-\nu)+(d) e^{I \lambda \Delta x}+(d+\nu) e^{-I \lambda \Delta x} \\
& =(-2 d-\nu)+d[\cos (\lambda \Delta x)+I \sin (\lambda \Delta x)]+(d+\nu)[\cos (\lambda \Delta x)-I \sin (\lambda \Delta x)] \\
& =(2 d+\nu)[\cos (\lambda \Delta x)-1]-\nu I \sin (\lambda \Delta x)
\end{aligned}
$$

If $d>=0$ and $\nu>=0$, the footprints are situated in the left half plane and the method is always stable. Thus, for constant material properties, the solution is found immediately, by taking a very large time step.

However, the region around the melting and solidification fronts have highly non-linear material properties. In this case, some instabilities can occur, de- 
pending on the method that is used to implement the latent heat of fusion. This will be discussed below.

\subsection{Latent heat of fusion}

There are different ways to take into account the effect of latent heat. $\mathrm{Hu}$ and Argyropoulos give an overview of different methods that can be used [9]. They state that solving the strong numerical solution, i.e. locating the exact moving boundary, is very difficult to apply for 3D cases. The alternative is to reformulate the problem in such a way that the Stefan condition ${ }^{3}$ is implicitly incorporated in a new form of equations, which applies over the entire region of a fixed domain. These methods are referred to as weak numerical solutions, in which explicit attention to the nature of the moving boundary is avoided. As such, eq. (4) can be solved for a temperature dependent $c^{\prime}\left(c_{a p p}\right.$ or $\left.c_{e f f}\right)$ or an extra heat sink $\dot{q}_{L_{f}}$. There are five main weak numerical solutions, which will be discussed here.

To evaluate different solutions, it is necessary to have a look at the stability and the convergence in such situation. This is discussed first.

\subsubsection{Stability and convergence}

For linear problems, there is a method to evaluate stability and convergence for a numerical method. However, for non-linear problems such a method is not available. The behaviour of a solution is validated by practical calculations. Convergence to the correct solution cannot be proved. Fortunately, a qualitative picture of the temperature field is known a priori. This enables us to comment on the stability of different implementations.

Consider for example the 1D problem, without heat source. The formulation of the problem can be written as

$$
\rho c^{\prime} V \frac{\partial T}{\partial x}=k \frac{\partial^{2} T}{\partial x^{2}}
$$

or

$$
\rho c V \frac{\partial T}{\partial x}=k \frac{\partial^{2} T}{\partial x^{2}}+\dot{q}_{L_{f}}
$$

From a physical point of view, latent heat can never cause the temperature field to have a local maximum or minimum. For a region where $c^{\prime}$ can be considered to be constant, only 4 solutions exist (see figure 6).

$\overline{{ }^{3} k_{s} \frac{\partial T_{s}}{\partial x}}-k_{l} \frac{\partial T_{l}}{\partial x}=L_{f} \rho \frac{d X}{d t}$, where $X$ is the position of the moving boundary between melt $l$ and solid $s$. 
$V<0$

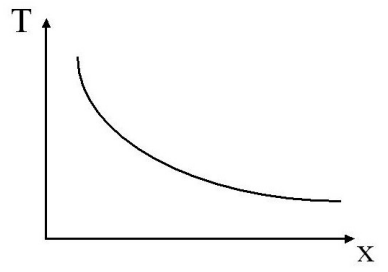

$T_{1}>T_{2}$

$V>0$

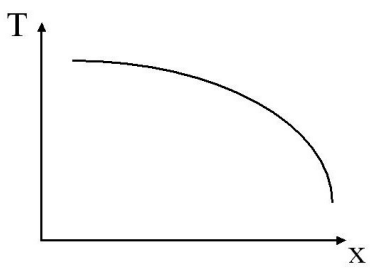

$T_{1}>T_{2}$

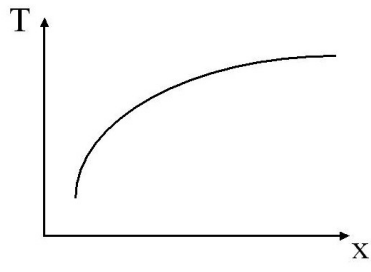

$T_{1}<T_{2}$

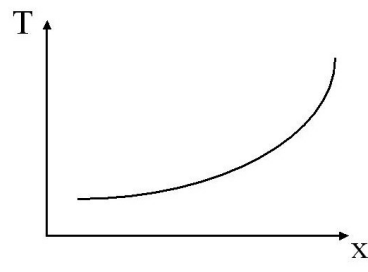

$T_{1}<T_{2}$

Fig. 6. Solutions of the 1D convection diffusion equation

Eq. (27) inherently satisfies this monotonous behaviour, while eq. (28) does not. This however, is not a prove for convergence or not. It only indicates that probably the convergence of a solution with $c^{\prime}$ will be faster.

The evaluation for the different solutions is based on mainly 3 observations. First, the monotonous behaviour must almost be satisfied. The reason is that for a non-monotonous temperature field after the heat source, the error on the location of the rear end of the melt pool becomes very large. This is a parameter of interest, since it determines the behaviour of the melt pool in different production processes. Next, the solidification area should have a temperature that is in the melting range. Otherwise, the solution is wrong. And last, this conditions must be met for relative large grid size. A 3D model suffers from the fact that it can become very large. Grid refinement is no option, because the solidification region is extended in three dimensions, and can be very long at the rear end for high Peclet numbers.

\subsubsection{Different solutions}

A first method is the apparent heat capacity method, where $c_{a p p}=c+c_{L_{f}}$ is calculated, with $L_{f}=\int_{T_{s}}^{T_{l}} c_{L_{f}} d T . T_{s}$ and $T_{l}$ are the solidus and liquidus temperature respectively. This definition results in a value for $c_{a p p}$ for each temperature, and does not imply a conservation of mass. The method is in- 
herent instable. A complete proof is out of the scope of this work. However, it can be shown that multiple solutions exist. Consider for example the one dimensional equation, without heat source ${ }^{4}$ :

$$
\theta_{i+1}-\theta_{i}\left(2+\frac{c_{a p p}\left(\theta_{i}\right) \rho V \Delta x}{k\left(\theta_{i}\right)}\right)+\theta_{i-1}\left(1+\frac{c_{a p p}\left(\theta_{i-1}\right) \rho V \Delta x}{k\left(\theta_{i-1}\right)}\right)=0
$$

Suppose that the boundary condition is $\theta=0.95$. The correct solution is a uniform temperature at this value. For $\theta_{i+1}=\theta_{i-1}=0.95$, two numerical solutions for the equation can be found: $\theta_{i, 1}=0.95$, which is what is expected, but also $\theta_{i, 2}$ slightly higher than 1 . The exact value depends on the temperature dependence of $c_{a p p}$, being a function of $L_{f}, T_{s}$ and $T_{l}$. Practically, there is no possibility to obtain a resulting temperature field that meets the criterion of monoticity.

The effective capacity method is a more stable (i.e. the resulting temperature field is closer to the correct temperature field) variant of the apparent heat capacity method. Here, the apparent heat capacity is averaged over the volume of a unite cell, supposing that the temperature profile between two cells is linear. However, there still is no conservation of energy, and practically, the resulting temperature field is not monotonous for grid sizes, that can be handled with Matlabß.

A third method is the heat integration method. The idea is that the temperature of the molten material will be lower by an amount of:

$$
\Delta T=L_{f} / c
$$

which corresponds to the extra energy for the phase transition itself. This energy cannot be used for overheating of the melt. For $\operatorname{TiAl}_{6} \mathrm{~V}_{4}, \Delta T$ is more than $500 \mathrm{~K}$.

However, for a 3D moving heat source, this method seems incorrect, The other methods indicate that the peak temperature is independent of $L_{f}$ for normal values of $L_{f}$. This can be understood by the fact that the net heat source, due to the latent heat of fusion, is zero, because both melting and solidification occur. This is only the case for a steady state solution, since the energy input is infinite. The energy consumed during melting is released during solidification.

Brockmann et al. [27] have described a similar problem for thin foils, with a surrounding gas. In this case the peak temperature will be much lower with latent heat, compared to the case without. In such case, the heat integration method could result in a better mathematical prediction of the temperature

\footnotetext{
$\overline{4}$ This is a special case of the general problem. If the solution of this problem is unstable, the solution of the more general problem will also be unstable. This is exactly what is observed.
} 
profile, compared to an analytical solution. However, the shape is not predicted in an appropriate way.

A fourth method is the source based method. Here a new source term is constructed:

$$
\dot{q}_{L_{f}}=-\rho \frac{\partial h_{L_{f}}}{\partial t}=-\rho V \frac{\partial \int c_{L_{f}} d T}{\partial x}
$$

Closely related to the source based method is the last method, the enthalpy method. Both are conservative ways to introduce the latent heat. The heat conduction equation can be written as

$$
\rho V \frac{\partial h}{\partial x}=\nabla \cdot(k(T) \nabla T)+\dot{q}
$$

This is implemented like the heat capacity method, with

$$
\bar{c}_{L_{f}}=\left(\int_{T_{1}}^{T_{2}} c_{L_{f}} d T\right) /\left(T_{2}-T_{1}\right)
$$

The effective heat capacity becomes

$$
c_{e f f}=c+\bar{c}_{L_{f}}
$$

The difference with the apparent heat capacity method is that here a change between $x_{i-1}$ and $x_{i}$ is observed, which guarantees the conservation of energy. Furthermore, for pure materials, the heat capacity becomes infinite in the heat capacity method. For the enthalpy method, this does not pose any numerical problem.

Mathematically, the enthalpy method and the source based method are equivalent:

$$
\dot{q}_{L_{f}}=-\rho V \frac{\partial \int c_{L_{f}} d T}{\partial x} \leftrightarrow-\rho V \frac{\partial \int c_{L_{f}} d T}{\partial x} \frac{\left(T_{2}^{t+1}-T_{1}^{t+1}\right)}{\left(T_{2}^{t}-T_{1}^{t}\right)}
$$

However, the stability is different. The enthalpy method is more stable as was shown in the section on stability and convergence. This results in an easier and faster convergence for the enthalpy method. The source based method can be made more stable, by implementing it implicitly, but this is somewhat less straightforward. As a result, the enthalpy method is selected for the implementation, and will be used in the remaining of the paper.

For the ease of calculation, we assume that heat capacity due to the latent heat is Gaussian. This assures a smooth transition. Hence we have

$$
c_{L_{f}}=\frac{L_{f}}{\sigma \cdot \sqrt{2 \pi}} \exp \left(-\frac{\left(T-T_{m}\right)^{2}}{2 \sigma^{2}}\right)
$$




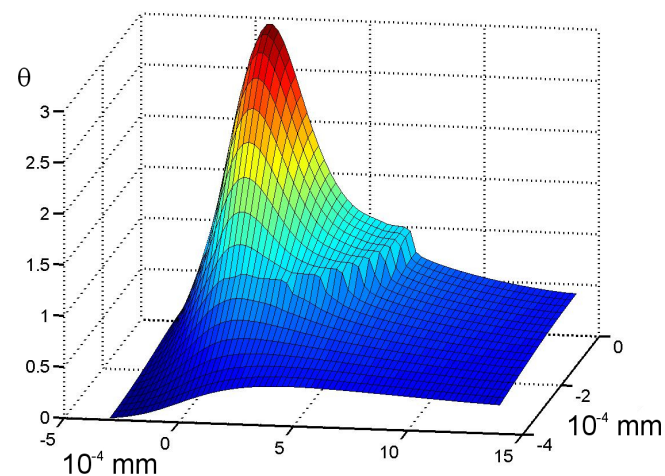

a. Latent heat modelled as $c_{L f}$

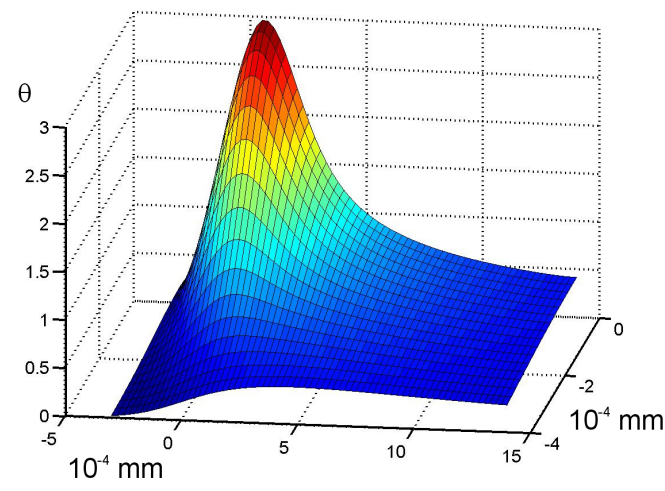

b. Without latent heat

Fig. 7. Temperature profile on the surface of a semi-infinite medium

Here, $\sigma$ is the standard deviation and is a measure of the melting region. It is calculated as $\sigma=a \cdot \Delta T_{m}$ where $a$ is a fixed, but in fact unknown, parameter.

Figure 7a a gives the result for a spot with $a_{h}=c_{h}=0.3 \mathrm{~mm}, V=0.05 \mathrm{~m} / \mathrm{s}$, $P_{L}=40 \mathrm{~W}$, rho $=4450 \mathrm{~kg} / \mathrm{m}^{3}, k=7 \mathrm{~W} / \mathrm{mK}, c=564 \mathrm{~J} / \mathrm{kgK}$ and a high $L_{f}=2920000 \mathrm{~J} / \mathrm{kg}(\Delta T=5 \mathrm{~K})$. This high value of $L_{f}$ was chosen for demonstration. For $\mathrm{TiAl}_{6} \mathrm{~V}_{4}, L_{f}=292000 \mathrm{~J} / \mathrm{kg}$. The effect for a smaller value of $L_{f}$ is rather small for a low Peclet number. All parameters are constant and the same for the liquid and the solid. In Figure $7 \mathrm{~b}, L_{f}=0$. One can verify that the temperature distribution only changes significantly at the rear end of the melt pool. The peak temperature is the same in both cases! The length of the melt pool can change significantly due to the latent heat of fusion for high Peclet numbers. A factor 2 or more is possible if scanning on a material with a low conductivity, like a powder bed.

If we want to determine the enthalpy $h_{L_{f}}$, we obtain

$$
\begin{aligned}
h_{L_{f}} & =\int_{0}^{T} c_{L_{f}} d T=\frac{L_{f}}{\sigma \cdot \sqrt{2 \pi}} \int_{0}^{T} e^{-\frac{\left(T-T_{m}\right)^{2}}{2 \sigma^{2}}} d T \\
& =\frac{L_{f}}{\sqrt{\pi}} \int_{-\frac{T m}{\sigma \sqrt{2}}}^{\frac{T-T_{m}}{\sigma \sqrt{2}}} e^{-x^{2}} d x \\
& =\frac{L_{f}}{2}\left[\operatorname{erf}\left(\frac{T-T_{m}}{\sigma \sqrt{2}}\right)-\operatorname{erf}\left(\frac{-T_{m}}{\sigma \sqrt{2}}\right)\right] \\
& \approx \frac{L_{f}}{2}\left[\operatorname{erf}\left(\frac{T-T_{m}}{\sigma \sqrt{2}}\right)+1\right]
\end{aligned}
$$


Hence we have

$$
\Delta_{1}^{2} h=\frac{L_{f}}{2}\left[\operatorname{erf}\left(\frac{T_{2}-T_{m}}{\sigma \sqrt{2}}\right)-\operatorname{erf}\left(\frac{T_{1}-T_{m}}{\sigma \sqrt{2}}\right)\right]
$$

The average power that is stored or released because of melting is

$$
P_{L_{f}}=\frac{\pi}{4} b d V L_{f} \rho
$$

where $\frac{\pi}{4} b d$ is the frontal area of the melt ( $b$ is the width and $d$ is the depth, and the surface is supposed to be elliptical).

For a Ti-6Al-4V alloy with $L_{f}=292000 \mathrm{~J} / \mathrm{kg}$ and $\rho=4450 \mathrm{~kg} / \mathrm{m}^{3}$, a spot of $0.3 \mathrm{~mm}$ travelling at a speed of $50 \mathrm{~mm} / \mathrm{s}$, we obtain, for $P_{L}=40 \mathrm{~W}$, a calculated width of the melt pool $b \approx 0.3 \mathrm{~mm}$ and a calculated depth of the melt pool $d \approx 0.2 \mathrm{~mm}$, so that $P_{L_{f}} \approx 3.06 \mathrm{~W}$. This is a small value compared to a normal power that is $P_{L}=50 \ldots 200 \mathrm{~W}$. The effect of the latent heat is therefore rather small. This is in agreement with the previous solution for the temperature field. The variation of the power of the heat source, or the change in absorptivity of the material might have the same size.

The same conclusion can be found when observing typical values for the melting efficiency. For Ti-6Al-4V, the relative importance of $L_{f}=292000 \mathrm{~J} / \mathrm{kg}$ is rather small, compared to the heating of the material, which is $c \Delta T \approx$ $564 \mathrm{~J} / \mathrm{kgK} * 1600 \mathrm{~K}=902400 \mathrm{~J} / \mathrm{kg}$. This means that only $25 \%$ of the useful energy is needed for the melting. If the melting efficiency is $\eta_{m} \approx 10 \%$, than proportion of the melting energy is only $2.5 \%$. This value compared to a laser power $P_{L}=100 \mathrm{~W}$, gives $2.5 \mathrm{~W}$.

\section{Conclusions}

For constant material properties, it is possible to solve the $3 \mathrm{D}$ heat conduction equation with a moving heat source analytically. This is elaborated for the case of a uniform heat source. The result is compared to existing solutions for the moving point source and a semi-ellipsoidal source. This analytical model has several advantages over FDM. First, it is very useful to study different heat source geometries. Moreover, with this analytical model, it is shown that the efficiency can be higher than 0.37, a value stated by Rykalin. Last but not least, analytical solution can serve very well as boundary condition for FDM. If the material parameters depend upon the temperature, as is especially the case when the effect of latent heat is to be studied, the problem can be solved with FDM. To implement the latent heat of fusion, the enthalpy method is most stable, converges fastest and incorporates the conservation of energy. 
For $\mathrm{TiAl}_{6} \mathrm{~V}_{4}$, the effect of the latent heat is rather small, except for the case when the conductivity is very low, like scanning in a loose powder bed. The implementation of the presented models results in a useful tool to investigate the effect of different processing parameters.

\section{Acknowledgement}

This research is supported by the K.U.Leuven Research Fund 'Geconcerteerde OnderzoeksActie' (GOA/2002/06), and the Belgian Science Policy through the projects 'Interuniversity Attraction Poles' (IAP P5/08) and 'Technological Attraction Poles' (TAP-32) for Rapid Manufacturing for Space Applications.

\section{References}

[1] P. W. Fuerschbach, G. R. Eisler, Determination of material properties for welding models by means of arc weld experiments, in: Sixth International Trends in Welding Research, Pine Mountain, Georgia, 2002, pp. 1 - 5.

[2] D. Rosenthal, Mathematical theory of heat distribution during welding and cutting, Welding Journal 20(5) (1941) 220 - 234.

[3] N. Rykalin, A. Uglov, A. Kokora, O. Glebov, Laser machining and welding, Moscow: Mir, 1978.

[4] H. Carslaw, J. Jaeger, Conduction of heat in solids, Oxford, 1990.

[5] T. Eager, N. Eager, Temperature fields produced by traveling distributed heat sources, Welding Journal 62(12) (1983) 346 - 355.

[6] J. Goldak, A. Chakravarti, M. Bibby, A double ellipsoid finite element model for welding heat sources (1985).

[7] N. T. Nguyen, A. Otha, K. Matsuoka, N. Suzuki, Y. Maeda, Analytic solutions for transient temperature of semi-infinite body subjected to 3-D moving heat sources, Welding Research Supplement (August 1999) 265 - 274.

[8] N. T. Nguyen, Y.-W. Mai, S. Simpson, A. Otha, Analytical approximate solution for double ellipsoidal heat source in finite thick plate, Welding Research (March 2004) $82-93$.

[9] H. Hu, S. A. Argyropoulos, Mathematical modelling of solidification and melting: a review, Modelling Simul. Mater. Sci. Eng. 4 (1996) $371-396$.

[10] M. Matsumoto, M. Shiomi, K. Osakada, F. Abe, Finite element analysis of single layer forming on metallic powder bed in rapid prototyping by selective laser processing, International Journal of Machine Tools \& Manufacture 42 (2002) $61-67$. 
[11] S. H. Choi, S. Samavedam, Modelling and optimisation of rapid prototyping, Computers in industry 47 (2002) $39-53$.

[12] T. Chen, Y. Zhang, Thermal modeling of metal powder-based selective laser sintering, in: Proc. Solid Freeform Fabrication, August 2005, pp. 356 - 369.

[13] A. V. Gusarov, J. P. Kruth, Mathematical simulation of radiation transfer in the powder bed: application to selective laser sintering, in: VRP International conference on advanced research in virtual and rapid prototyping, 2003, pp. 241 $-248$.

[14] R. F. Gould, Contact angle: wettability and adhesion, American Chemical Society, 1964.

[15] R. V. Roy, L. W. Schwartz, On the stability of liquid ridges, Journal on Fluid Mechanics 391 (1999) $293-318$.

[16] G. N. Levy, R. Schindel, J. P. Kruth, Rapid manufacturing and rapid tooling with layer manufacturing $(\mathrm{lm})$ technologies, state of the art and future perspectives, CIRP Annals 52/2 (2003) 589.

[17] J. P. Kruth, P. Mercelis, J. Van Vaerenbergh, L. Froyen, M. Rombouts, Binding mechanisms in selective laser sintering and selective laser melting, Rapid Prototyping Journal 11 (1) (January 2005) 26 - 36.

[18] N. Christensen, V. Davies, K. Gjermundsen, The distribution of temperature in arc welding, British Welding Journal 12(2) (1965) $54-75$.

[19] J. K. Carson, S. J. Lovatt, D. J. Tanner, A. C. Cleland, Thermal conductivity bounds for isotropic, porous materials, Int. Journal of Heat and Mass Transfer 48 (2005) $2150-2158$.

[20] W. Meiners, Direktes selektives laser sintern einkomponentiger metallischer werkstoffe, Ph.D. thesis, Aachen (1999).

[21] J. C. Y. Koh, A. Fortini, Prediction of thermal conductivity and electrical resistivity of porous metallic materials, Int. Journal of Heat and Mass Transfer 16 (1973) $2013-2021$.

[22] A. V. Gusarov, T. Laoui, L. Froyen, V. I. Titov, Contact thermal conductivity of a powder bed in selective laser sintering, Int. Journal of Heat and Mass Transfer 46 (2003) 1103 - 1109.

[23] J. F. Ready, D. F. Farson (Eds.), Handbook of laser materials processing, Laser Institute of America, 2001.

[24] M. Pietro, C. Rivela, B. Marco, Mathematical modelling of laser treatment processes, Laser Materials Processing: industrial and microelectronics applications SPIE 2207 (1994) $256-268$.

[25] D. F. De Lange, S. Postma, J. Meijer, Modelling and observation of laser welding: The effect of latent heat, in: Proceedings of ICALEO 2003, Vol. Section C, Jacksonville FL, 2003, pp. $154-162$. 
[26] C. Hirsch, Numerical computation of internal and external flows, Vol. 1, John Wiley \& Sons, 1995.

[27] R. Brockmann, K. Dickmann, P. Geshev, K.-J. Matthes, Calculation of laserinduced temperature field on moving thin metal foils in consideration of stefan problem, Optics \& Laser Technology 35 (2003) 115 - 122. 